\title{
Genetic variations associated with telomere length confer risk of gastric cardia adenocarcinoma
}

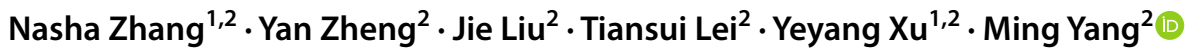

Received: 18 December 2018 / Accepted: 11 March 2019 / Published online: 21 March 2019

(c) The International Gastric Cancer Association and The Japanese Gastric Cancer Association 2019

\begin{abstract}
Background Aberrant telomere lengthening is a critical feature of malignant cells. Short leukocyte telomere length (LTL) confers elevated risk of gastric cardia adenocarcinoma (GCA). Multiple genome-wide association studies (GWAS) identified various single-nucleotide polymorphisms (SNPs) associated with LTL in different ethnic populations. However, it remains largely unexplored how these genetic variants are involved in GCA susceptibility.

Methods We systematically screened GWAS-identified candidate SNPs and tested the impact of 30 polymorphisms in genes associated with interindividual LTL variation on GCA using two-stage case-control comparisons consisting of 1024 GCA patients and 1118 controls.

Results We observed that CXCR4 rs6430612, TERT rs10069690, and rs2853676 as well as VPS34 rs2162440 are significantly associated with GCA development. A 0.64-fold decreased risk of GCA is associated with the CXCR4 rs6430612 CT genotype compared with the CC genotype $(P=0.002)$. On the contrary, the TERT rs 10069690 TT genotype carriers had a 1.83-fold increased risk to develop GCA compared to the CC genotype carriers $\left(P=5.8 \times 10^{-6}\right)$. We also detected a 2.17-fold increased OR for GCA that was associated with the TERT rs2853676 TT genotype $\left(P=2.6 \times 10^{-6}\right)$. In addition, the odds of having the VPS34 rs2162440 GA genotype in GCA patients were 1.35 compared with the GG genotype $(P=0.002)$. In stratified analyses, the association between TERT rs10069690 polymorphism and GCA was more pronounced in nonsmokers $\left(P_{\text {interaction }}=9.7 \times 10^{-5}\right)$ and nondrinkers $\left(P_{\text {interaction }}=4.6 \times 10^{-5}\right)$.
\end{abstract}

Conclusions Our results highlight the importance of both LTL and LTL-related genetic variants to GCA predisposition.

Keywords Telomere $\cdot$ GCA $\cdot$ CXCR4 $\cdot$ TERT $\cdot$ VPS34 $\cdot$ Genetic polymorphism

\section{Introduction}

Estimated 951,600 new gastric cancer cases and 723,100 deaths occurred in 2012 worldwide [1]. Gastric cancer incidences vary widely across countries, and in general, incidence rates are highest in Eastern Asia, Central and Eastern

Electronic supplementary material The online version of this article (https://doi.org/10.1007/s10120-019-00954-8) contains supplementary material, which is available to authorized users.

Ming Yang

aaryoung@yeah.net; yangm@sdu.edu.cn

1 Cheeloo College of Medicine, Shandong University, Jinan, Shandong, China

2 Shandong Provincial Key Laboratory of Radiation Oncology, Cancer Research Center, Shandong Cancer Hospital Affiliated to Shandong University, Shandong Academy of Medical Sciences, Jinan 250117, Shandong, China
Europe, and South America, and lowest in Northern America and most parts of Africa [1]. According to National Central Cancer Registry of China (NCCRC) updated nationwide statistics of cancer incidence and mortality in China using population-based cancer registration data in 2013, gastric cancer was the second most common cancer, accounting for about $11.6 \%(427,100)$ of all new cancers [2] and the third most common cause of cancer death $(301,200)$ of all cancer deaths in China [2]. Gastric cancer has two main subtypes including cardia (proximal, gastroesophageal junction) and noncardia (fundus, body, distal, and lesser or greater curvature). Gastric cardia carcinoma (GCA) commonly occurs in the $1 \mathrm{~cm}$ proximal and $2 \mathrm{~cm}$ distal region of the esophagogastric junction. Using Cancer Incidence in Five Continents Volume X, it was estimated that there were 260,000 cases of GCA (age standardized incidence rates: 3.3 per 100,000) worldwide in 2012 [3]. Dissimilar to gastric cancer at other sites, GCA is a special type of gastric cancer having its own 
epidemiological characteristics, etiology, pathogenesis, and clinical manifestations [4]. Cigarette smoking, heavy alcohol consumption, dietary carcinogen exposure, low-intake of fruits and vegetables, and gastroesophageal reflux disease have been identified as GCA environmental risk factors [5-8]. Interestingly, accumulated evidences demonstrated that genetic makeups also play a part in etiology of GCA [9-12].

As nucleoprotein structures capping and protecting the ends of chromosomes, telomeres are engaged in a host of cellular functions $[13,14]$. Telomeres shorten with each cell division and leucocyte telomere length has been shown to decrease with age at a rate of 20-40 base pairs (bp) per year $[15,16]$. Leucocyte telomere length is a predictor for a number of common age-related diseases including cancers [17]. Consistent to these findings, we previously showed that short leucocyte telomere length contributes to increased susceptibility to GCA [10]. Telomeres length is a heritable trait, with heritability ranging from 44 to $80 \%$ and regulated by multiple genes [18, 19]. Several genome-wide association studies (GWAS) and candidate gene studies identified dozens of single-nucleotide polymorphisms (SNPs) associated with leucocyte telomere length in different ethnic populations [20-29]. Intriguingly, multiple telomere length-related SNPs confer risk of different malignancies, such as prostate cancer, ovarian cancer, leukemia, colorectal cancer, glioma, and pancreatic cancer. Nevertheless, the mechanisms by which common variants associated with leucocyte telomere length impact development of GCA are still unclear.

In view of the importance of telomeres in carcinogenesis, we hypothesized that the aforementioned telomere length-related genetic polymorphisms may contribute to GCA risk in Chinese populations. To test this hypothesis, we conducted a large case-control study of GCA with two Chinese populations from different regions of China.

\section{Materials and methods}

\section{Study case-control sets}

This study consisted of two case-control sets (Table 1): (a) Shandong set (discovery set): 584 patients with GCA from Shandong Cancer Hospital affiliated to Shandong University (Jinan, Shandong Province, China) and sex- and agematched ( \pm 5 years) 568 controls. Patients were recruited between August 2012 and January 2018 at Shandong Cancer Hospital. Exclusion criteria were that patients with a second primary tumor or the primary tumor outside of gastric cardia. Control subjects were randomly selected from a pool of 6800 individuals from a comprehensive physical examination conducted in Jinan city and the surrounding areas during the same time period as the patients were collected. Part of the case-control set has been reported previously [10]. (b) Jiangsu set (validation set): 440 GCA patients from Huaian No. 2 Hospital (Huaian, Jiangsu Province, China) and sexand age-matched 500 controls. Patients were consecutively recruited between June 2010 and January 2018 at Huaian No. 2 Hospital. The controls were cancer-free individuals based on a physical examination, randomly selected from a pool of 4000 subjects which were recruited from the same hospital during the same time period as the patients were
Table 1 Distribution of selected characteristics among GCA cases and controls

\begin{tabular}{|c|c|c|c|c|c|c|}
\hline \multirow[t]{4}{*}{ Variables } & \multicolumn{3}{|c|}{ Shandong set (discovery set) } & \multicolumn{3}{|c|}{ Jiangsu set (validation set) } \\
\hline & Cases & Controls & $P^{\mathrm{a}}$ & Cases & Controls & $P^{\mathrm{a}}$ \\
\hline & No. $(\%)$ & No. $(\%)$ & & No. $(\%)$ & No. $(\%)$ & \\
\hline & $n=584$ & $n=568$ & & $n=440$ & $n=500$ & \\
\hline Age (year) & & & 0.458 & & & 0.320 \\
\hline$\leq 66$ & $314(53.8)$ & $293(51.6)$ & & $226(51.4)$ & $265(48.2)$ & \\
\hline$>66$ & $270(46.2)$ & $275(48.4)$ & & $214(48.6)$ & $285(51.8)$ & \\
\hline Sex & & & 0.598 & & & 0.512 \\
\hline Male & $494(84.6)$ & $474(83.5)$ & & $367(83.4)$ & $450(81.8)$ & \\
\hline Female & $90(15.4)$ & $94(16.5)$ & & $73(16.6)$ & $100(18.2)$ & \\
\hline Smoking status & & & $<0.001$ & & & $<0.001$ \\
\hline No & $309(52.9)$ & $449(79.0)$ & & $213(48.4)$ & $412(74.9)$ & \\
\hline Yes & $275(47.1)$ & $119(21.0)$ & & $227(51.6)$ & $138(25.1)$ & \\
\hline Drinking status & & & 0.006 & & & 0.005 \\
\hline No & $349(59.8)$ & $384(97.6)$ & & $238(54.1)$ & $346(62.9)$ & \\
\hline Yes & $235(40.2)$ & 184 (32.4) & & $202(45.9)$ & $204(37.1)$ & \\
\hline
\end{tabular}

GCA gastric cardia adenocarcinoma

${ }^{\text {a }}$ Two-sided Chi-square test 
collected. The diagnosis of all patients was histologically confirmed. Individuals who smoked one cigarette per day for over 1 year were considered as smokers. Subjects were considered as alcohol drinkers, if they drank at least once per week. All subjects were ethnic Han Chinese. At recruitment, informed consent was obtained from each subject and each participant was then interviewed to collect detailed information on demographic characteristics, such as sex, age, cigarette smoking and alcohol drinking. This study was approved by the institutional Review Boards.

\section{Genetic polymorphism selection and genotyping}

After literature searches with search terms of "telomere length", "polymorphism", "variant", "SNP", as well as their combinations, we identified 88 SNPs significantly associated with telomere length published before April 2018. By systematically screening CHB (Chinese Han Beijing) and CHS (Han Chinese South) data of the 1000 genomes project, we excluded 53 SNPs which either have minor allele frequencies $(\mathrm{MAF})<0.03$ in Chinese populations or show strong linkage disequilibrium with other candidate SNP(s) with an $r^{2}$ threshold of 0.80 . Five polymorphisms were excluded, since they are unable to be genotyped using the iPLEX Sequenom MassARRAY platform (Sequenom Inc., San Diego, CA, USA). Finally, a total of 30 candidate genetic variants were included in the study (Table 1).

Thirty telomere length-related SNPs were first analyzed in the Shandong case-control set (discovery set). CXCR4 rs6430612, TERT rs 10069690 and rs2853676, and VPS34 rs 2162440 were then examined in the Jiangsu case-control validation set. Genotypes of all these polymorphisms were examined using the MassARRAY platform as previously reported [30-32]. A 15\% random sample was reciprocally tested and the reproducibility was $100 \%$.

\section{Statistics}

Pearson's chi-square test was used to examine the differences in demographic variables, smoking status, drinking status and genotype distributions of all 30 SNPs between GCA cases and controls. The associations between telomere length-related SNPs and GCA risk were estimated by odds ratios (ORs) and their 95\% confidence intervals (95\% CIs) computed by logistic regression models. All ORs were adjusted for age, sex, smoking or drinking status, where it was appropriate. The association of SNPs with telomere length was assessed using linear regression adjusted for age, sex, smoking and drinking status. During meta-analyses, a fixed effect model (the Mantel-Haenszel method) was performed to calculate the combined OR using Stata Statistical package (version 11.0; Stata Corp.). Bonferroni correction was used for multiple comparisons. All statistical tests were two-sided and were performed using SPSS 16.0 (SPSS Inc.).

\section{Results}

\section{Subject characteristics}

As shown in Table 1, age and sex distributions were adequately matched in either Shandong set or Jiangsu set. There were no significant statistical differences in the distributions of median age and sex between GCA patients and controls in both case-control sets (all $P>0.05$ ). However, smokers and drinkers accounted for a higher proportion among GCA patients compared with those in controls (Shandong set: smokers: $47.1 \%$ vs. $21.0 \%, P<0.001$; drinkers: $40.2 \%$ vs. $32.4 \%, P=0.006$; Jiangsu set: smokers: $51.67 \%$ vs. $25.1 \%$, $P<0.001$; drinkers: $45.9 \%$ vs. $37.1 \%, P=0.005$ ) (Table 1 ).

\section{Allelic frequencies of $\mathbf{3 0}$ telomere length-related SNPs in the discovery set}

Allele frequencies of 30 telomere length-related genetic polymorphisms in cases and controls are showed in Table 2. All observed genotype frequencies in both controls and patients conform to Hardy-Weinberg equilibrium. Distributions of the genotypes were then compared among cases and controls. Frequencies of CXCR4 rs6430612 C and T alleles among patients differed significantly from those among controls $\left(\chi^{2}=7.74, P=0.005\right)$, with the frequency of $\mathrm{T}$ allele being significantly lower among patients than among controls (4.6\% vs. 7.4\%). On the contrary, allele frequencies of the TERT rs 10069690 and rs2853676 as well as VPS34 rs2162440 genetic variants were significantly different among cases and controls (rs10069690: $\chi^{2}=7.11$, $P=0.008$; rs 2853676: $\chi^{2}=18.65, P=1.6 \times 10^{-5}$; rs 2162440 : $\chi^{2}=4.21, P=0.040$ ), with the frequency of the rs 10069690 $\mathrm{T}$, rs2853676 $\mathrm{T}$ as well as rs2162440 A allele being higher in cases than in controls (rs10069690: $21.2 \%$ vs. $16.7 \%$; rs2853676: $19.8 \%$ vs. $12.9 \%$; rs2162440: $22.6 \%$ vs. $19.1 \%$ ). However, no statistically significant allele differences of other SNPs were observed between GCA cases and controls (all $P>0.05$ ) (Table 2).

\section{Association between telomere length-related polymorphisms and GCA risk in both discovery and validation case-control sets}

Unconditional logistic regression analysis was used to estimate associations between genotypes of telomere length-related CXCR4 rs6430612, TERT rs10069690 and rs2853676, and VPS34 rs2162440 polymorphisms and GCA risk in Shandong set (Table 3). The CXCR4 rs6430612 T 
Table 2 Association between GCA susceptibility and telomere length-related genetic variations from previously published studies (Shandong discovery set)

\begin{tabular}{|c|c|c|c|c|c|c|c|c|}
\hline Genes & SNP IDs & Location $^{\mathrm{a}}$ & Alleles $^{\mathrm{b}}$ & MAF of cases/controls & $\mathrm{OR}^{\mathrm{c}}$ & $95 \% \mathrm{CI}^{\mathrm{c}}$ & $P^{\mathrm{c}}$ & $\begin{array}{l}\text { Effect allele }{ }^{\mathrm{d}} \text { and } \\
\text { reference }\end{array}$ \\
\hline$A C Y P 2$ & rs 11125529 & $\begin{array}{l}\text { Chromo- } \\
\text { some 2:54475866 }\end{array}$ & $\mathrm{C} / \mathrm{A}$ & $0.116 / 0.123$ & 0.94 & $0.74-1.21$ & 0.631 & A; Ojha et al. [20] \\
\hline DDX18 & rs6712766 & $\begin{array}{l}\text { Chromo- } \\
\text { some 2:118611789 }\end{array}$ & $\mathrm{G} / \mathrm{A}$ & $0.049 / 0.053$ & 0.91 & $0.63-1.32$ & 0.628 & A; Julin et al. [21] \\
\hline CXCR4 & rs6430612 & $\begin{array}{l}\text { Chromo- } \\
\text { some 2:137006198 }\end{array}$ & $\mathrm{C} / \mathrm{T}$ & $0.046 / 0.074$ & 0.62 & $0.43-0.87$ & 0.006 & T; Levy et al. [22] \\
\hline TERC & rs12696304 & $\begin{array}{l}\text { Chromo- } \\
\text { some 3:169481271 }\end{array}$ & $\mathrm{G} / \mathrm{C}$ & $0.299 / 0.289$ & 1.05 & $0.88-1.25$ & 0.619 & G; Shen et al. [23] \\
\hline TERC & rs 10936599 & $\begin{array}{l}\text { Chromo- } \\
\text { some 3:169492101 }\end{array}$ & $\mathrm{T} / \mathrm{C}$ & $0.448 / 0.453$ & 0.98 & $0.83-1.16$ & 0.829 & T; Walsh et al. [29] \\
\hline TERC & rs 16847897 & $\begin{array}{l}\text { Chromo- } \\
\text { some 3:169568116 }\end{array}$ & $\mathrm{G} / \mathrm{C}$ & $0.379 / 0.394$ & 0.94 & $0.80-1.11$ & 0.463 & G; Codd et al. [26] \\
\hline TERC & rs1920116 & $\begin{array}{l}\text { Chromo- } \\
\text { some 3:169579971 }\end{array}$ & G/A & $0.424 / 0.411$ & 1.05 & $0.89-1.24$ & 0.532 & A; Jones et al. [24] \\
\hline$T E R T$ & rs4246742 & $\begin{array}{l}\text { Chromo- } \\
\text { some 5:1267356 }\end{array}$ & $\mathrm{T} / \mathrm{A}$ & $0.318 / 0.333$ & 0.93 & $0.79-1.11$ & 0.422 & T; Terry et al. [25] \\
\hline TERT & rs 13172201 & $\begin{array}{l}\text { Chromo- } \\
\text { some 5:1271661 }\end{array}$ & $\mathrm{C} / \mathrm{T}$ & $0.146 / 0.145$ & 1.01 & $0.79-1.27$ & 0.969 & T; Bao et al. [36] \\
\hline TERT & rs 10069690 & $\begin{array}{l}\text { Chromo- } \\
\text { some 5:1279790 }\end{array}$ & $\mathrm{C} / \mathrm{T}$ & $0.212 / 0.167$ & 1.35 & $1.10-1.67$ & 0.005 & C; Julin et al. [21] \\
\hline TERT & rs 2736100 & $\begin{array}{l}\text { Chromo- } \\
\text { some 5:1286516 }\end{array}$ & $\mathrm{C} / \mathrm{A}$ & $0.418 / 0.412$ & 1.01 & $0.86-1.20$ & 0.872 & C; Ojha et al. [20] \\
\hline TERT & rs2853677 & $\begin{array}{l}\text { Chromo- } \\
\text { some 5:1287194 }\end{array}$ & $\mathrm{A} / \mathrm{G}$ & $0.355 / 0.362$ & 0.97 & $0.82-1.15$ & 0.728 & G; Bao et al. [36] \\
\hline$T E R T$ & rs2853676 & $\begin{array}{l}\text { Chromo- } \\
\text { some 5:1288547 }\end{array}$ & $\mathrm{C} / \mathrm{T}$ & $0.198 / 0.129$ & 1.65 & $1.32-2.08$ & $1.6 \times 10^{-5}$ & C; Julin et al. [21] \\
\hline$T E R T$ & rs2736098 & $\begin{array}{l}\text { Chromo- } \\
\text { some 5:1294086 }\end{array}$ & $\mathrm{C} / \mathrm{T}$ & $0.344 / 0.343$ & 0.99 & $0.83-1.19$ & 0.937 & T; Julin et al. [21] \\
\hline TERT & rs 451360 & $\begin{array}{l}\text { Chromo- } \\
\text { some 5:1319680 }\end{array}$ & $\mathrm{C} / \mathrm{A}$ & 0.095/0.089 & 1.08 & $0.81-1.43$ & 0.622 & C; Bao et al. [36] \\
\hline$T E R T$ & rs 402710 & $\begin{array}{l}\text { Chromo- } \\
\text { some 5:1320722 }\end{array}$ & $\mathrm{C} / \mathrm{T}$ & $0.319 / 0.316$ & 1.03 & $0.86-1.23$ & 0.774 & C; Bao et al. [36] \\
\hline CLPTM1L & rs401681 & $\begin{array}{l}\text { Chromo- } \\
\text { some 5:1322087 }\end{array}$ & $\mathrm{C} / \mathrm{T}$ & $0.339 / 0.333$ & 1.03 & $0.87-1.24$ & 0.711 & T; Julin et al. [21] \\
\hline SLC44A4 & rs2736428 & $\begin{array}{l}\text { Chromo- } \\
\text { some 6:31843924 }\end{array}$ & $\mathrm{C} / \mathrm{T}$ & $0.333 / 0.324$ & 1.03 & $0.87-1.23$ & 0.705 & C; Levy et al. [22] \\
\hline LOC105376013 & rs 10511887 & $\begin{array}{l}\text { Chromo- } \\
\text { some 9:31837334 }\end{array}$ & $\mathrm{A} / \mathrm{G}$ & $0.268 / 0.255$ & 1.06 & $0.88-1.28$ & 0.536 & A; Codd et al. [26] \\
\hline$O B F C 1$ & rs 10883943 & $\begin{array}{l}\text { Chromosome } \\
\text { 10:105651416 }\end{array}$ & $\mathrm{G} / \mathrm{T}$ & $0.327 / 0.303$ & 1.14 & $0.94-1.36$ & 0.177 & G; Julin et al. [21] \\
\hline$O B F C 1$ & rs4387287 & $\begin{array}{l}\text { Chromo- } \\
\text { some 10:105677897 }\end{array}$ & $\mathrm{A} / \mathrm{C}$ & $0.137 / 0.154$ & 0.88 & $0.70-1.10$ & 0.263 & A; Levy et al. [22] \\
\hline GRIA4 & rs610160 & $\begin{array}{l}\text { Chromo- } \\
\text { some 11:105696895 }\end{array}$ & $\mathrm{T} / \mathrm{C}$ & $0.162 / 0.167$ & 0.97 & $0.77-1.21$ & 0.780 & C; Codd et al. [26] \\
\hline$C S N K 2 A 2$ & rs74019828 & $\begin{array}{l}\text { Chromo- } \\
\text { some 16:58209274 }\end{array}$ & G/A & $0.089 / 0.111$ & 0.87 & $0.66-1.14$ & 0.301 & A; Julin et al. [21] \\
\hline MPHOSPH6 & rs2967374 & $\begin{array}{l}\text { Chromo- } \\
\text { some 16:82209861 }\end{array}$ & G/A & $0.235 / 0.268$ & 0.83 & $0.68-1.01$ & 0.065 & A; Julin et al. [21] \\
\hline CTC1 & rs3027234 & $\begin{array}{l}\text { Chromo- } \\
\text { some 17:8136092 }\end{array}$ & $\mathrm{C} / \mathrm{T}$ & $0.041 / 0.042$ & 0.98 & $0.65-1.48$ & 0.918 & C; Julin et al. [21] \\
\hline$M E O X 1$ & rs8081000 & $\begin{array}{l}\text { Chromo- } \\
\text { some 17:41715233 }\end{array}$ & $\mathrm{A} / \mathrm{G}$ & $0.414 / 0.390$ & 1.10 & $0.93-1.29$ & 0.282 & A; Levy et al. [22] \\
\hline
\end{tabular}


Table 2 (continued)

\begin{tabular}{llllllllll}
\hline Genes & SNP IDs & Location $^{\mathrm{a}}$ & Alleles $^{\mathrm{b}}$ & MAF of cases/controls & OR $^{\mathrm{c}}$ & 95\% $\mathrm{CI}^{\mathrm{c}}$ & $P^{\mathrm{c}}$ & $\begin{array}{c}\text { Effect allele }^{\mathrm{d}} \text { and } \\
\text { reference }\end{array}$ \\
\hline VPS34 & rs2162440 & $\begin{array}{c}\text { Chromo- } \\
\text { some 18:35214006 }\end{array}$ & G/A & $0.226 / 0.191$ & 1.23 & $1.01-1.51$ & 0.046 & G; Mangino et al. [27] \\
CCBE1 & rs1791285 & $\begin{array}{c}\text { Chromo- } \\
\text { some 18:57366183 }\end{array}$ & T/C & $0.080 / 0.081$ & 0.99 & $0.74-1.34$ & 0.986 & C; Julin et al. [21] \\
ADA & rs73598374 & $\begin{array}{c}\text { Chromo- } \\
\text { some 20:43280227 }\end{array}$ & C/T & $0.023 / 0.019$ & 1.23 & $0.71-2.15$ & 0.466 & C; Concetti et al. [28] \\
RTEL1 & rs6010620 & $\begin{array}{c}\text { Chromo- } \\
\text { some 20:62309839 }\end{array}$ & A/G & $0.267 / 0.236$ & 1.18 & $0.98-1.43$ & 0.088 & A; Walsh et al. [29] \\
\hline
\end{tabular}

GCA gastric cardia adenocarcinoma, $S N P$ single-nucleotide polymorphism, $M A F$ minor allele frequency, $O R$ odds ratio, $C I$ confidence interval ${ }^{\mathrm{a}}$ Reference genome GRCh37.p13

${ }^{\mathrm{b}}$ Major allele/minor allele for each polymorphism

${ }^{\mathrm{c}}$ Data were calculated by logistic regression with adjustment for age, sex, smoking and drinking status

${ }^{\mathrm{d}}$ The effect allele possibly associated with increased leukocyte telomere length

allele was showed to be protective allele; subjects having the CT genotype had an OR of 0.66 (95\% CI 0.45-0.96; $P=0.029)$ for developing GCA compared with subjects having the $\mathrm{CC}$ genotype. It was observed that a significantly increased risk of developing GCA was associated with the TERT rs 10069690 TT genotype (OR 1.69; 95\% CI $1.20-2.37 ; P=0.003)$ compared with the rs $10069690 \mathrm{CC}$ genotype. However, the TERT rs10069690 CT heterozygous genotype showed no effect on GCA risk. In addition, individuals with the TERT rs $2853676 \mathrm{CT}$ or TT genotype also showed significantly increased GCA risk compared with those with the rs2853676 CC genotype (OR 1.50; 95\% CI 1.15-1.95; $P=0.003$; OR 2.20; 95\% CI 1.41-3.45; $P=5.8 \times 10^{-4}$ ) (Table 3$)$. A significantly increased OR was associated with the VPS34 rs2162440 GA genotype (OR $1.31,95 \%$ CI 1.02-1.68, $P=0.037)$, but was not associated with the rs 2162440 AA genotype $(P=0.348)$. All ORs were adjusted for sex, age, smoking and alcohol drinking status. We also analyzed the association of 30 SNPs with telomere length in cases and controls of Shandong set. As shown in Supplementary Table 1, we found that CXCR4 rs6430612, TERT rs10069690, and rs2853676 as well as VPS34 rs2162440 polymorphisms are significantly associated with telomere length. In detail, the CXCR4 rs6430612 T allele was associated with long telomere length and showed to be protective allele. On the contrary, the minor alleles of TERT rs 10069690 and rs2853676 as well as VPS34 rs2162440 are all associated with short telomere length. However, we did not find significant correlation between polymorphisms and telomere length in controls. Similar association trends between SNPs and telomere length in controls exist as it does among GCA cases (Supplementary Table 2).

In the Jiangsu validation set, the significant associations between CXCR4 rs6430612, TERT rs 10069690 and rs2853676, and VPS34 rs2162440 polymorphisms and
GCA risk were also observed (Table 3). Logistic regression analyses revealed that individuals with CXCR4 rs6430612 CT genotype were significantly associated with decreased GCA risk (OR 0.60, 95\% CI 0.38-0.94, $P=0.025)$. Carriers of the TERT rs 10069690 TT genotype showed significantly elevated risk to develop GCA compared with the rs 10069690 CC carriers (OR 2.02, 95\% CI 1.34-3.04, $\left.P=8.0 \times 10^{-4}\right)$. Similarly, significantly increased GCA risk was observed among TERT rs2853676 CT or TT carriers compared to individuals with the s2853676 CC genotype (OR 1.76, 95\% CI 1.31-2.35, $P=1.6 \times 10^{-4}$; OR $2.12,95 \%$ CI $1.32-3.38, P=0.002$ ). Moreover, the VPS34 rs2162440 GA genotype was also associated with 1.40 -fold (95\% CI $1.07-1.84 ; P=0.015$ ) elevated GCA risk. However, this association was not statistically significant for the rs2162440 AA genotype $(P=0.211)$.

In the pooled analyses, we found that reduced GCA risk was associated with the CXCR4 rs6430612 CT genotype (OR $0.64,95 \%$ CI $0.78-0.85, P=0.002$ ) (Table 3 ). The TERT rs10069690 CT or TT genotype carriers had a 1.24fold or 1.83-fold increased risk to develop GCA compared to the rs 10069690 CC genotype carriers (95\% CI 1.03-1.50, $P=0.023$ or $95 \%$ CI $1.41-2.38, P=5.8 \times 10^{-6}$ ). The TERT rs $2853676 \mathrm{CT}$ or TT genotype was also significantly associated with elevated GCA risk (OR 1.62, 95\% CI 1.33-1.97, $P=1.3 \times 10^{-6}$; OR $2.17,95 \%$ CI $1.57-3.00, P=2.6 \times 10^{-6}$ ) compared to the rs $2853676 \mathrm{CC}$ genotype. In addition, the odds of having the VPS34 rs2162440 GA genotype in GCA patients was 1.35 (95\% CI 1.12-1.62, $P=0.002)$ compared with the rs 2162440 GG genotype. As shown in Supplementary Fig. 1, meta-analyses demonstrated that all four SNPs also significantly contributed to GCA risk (CXCR4 rs6430612: OR 0.62, 95\% CI 0.47-0.83; TERT rs10069690: OR 1.36, 95\% CI 1.14-1.63; TERT rs2853676: OR 1.75, 
Table 3 Genotype frequencies of CXCR4, TERT and VPS34 SNPs among cases and controls and their association with GCA risk

\begin{tabular}{|c|c|c|c|c|c|c|c|c|c|}
\hline \multirow{2}{*}{ Genes } & \multirow[t]{2}{*}{ SNP IDs } & \multicolumn{4}{|l|}{ Shandong set } & \multicolumn{4}{|l|}{ Jiangsu set } \\
\hline & & Cases, no. (\%) & $\begin{array}{l}\text { Controls, no. } \\
(\%)\end{array}$ & $\mathrm{OR}^{1}(95 \% \mathrm{CI})$ & $P$ & Cases, no. (\%) & $\begin{array}{l}\text { Controls, no. } \\
(\%)\end{array}$ & $\mathrm{OR}^{1}(95 \% \mathrm{CI})$ & $P$ \\
\hline \multirow[t]{6}{*}{ CXCR4 } & rs6430612 & $n=582$ & $n=568$ & & & $n=438$ & $n=550$ & & \\
\hline & $\mathrm{CC}$ & $530(91.0)$ & $490(86.3)$ & $\begin{array}{l}1.00 \text { (refer- } \\
\text { ence) }\end{array}$ & & $406(92.7)$ & 482 (87.6) & $\begin{array}{l}1.00 \text { (refer- } \\
\text { ence) }\end{array}$ & \\
\hline & $\mathrm{CT}$ & $51(8.8)$ & $72(12.7)$ & $\begin{array}{l}0.66(0.45- \\
0.96)\end{array}$ & 0.029 & $32(7.3)$ & $63(11.5)$ & $\begin{array}{l}0.60(0.38- \\
0.94)\end{array}$ & 0.025 \\
\hline & $\mathrm{TT}$ & $1(0.2)$ & $6(1.0)$ & $\mathrm{NC}$ & $\mathrm{NC}$ & $0(0.0)$ & $5(0.9)$ & $\mathrm{NC}$ & $\mathrm{NC}$ \\
\hline & $\mathrm{C}$ allele & & & $\begin{array}{l}1.00 \text { (refer- } \\
\text { ence) }\end{array}$ & & & & $\begin{array}{l}1.00 \text { (refer- } \\
\text { ence) }\end{array}$ & \\
\hline & T allele & & & $\begin{array}{l}0.62(0.43- \\
0.87)\end{array}$ & 0.006 & & & $\begin{array}{l}0.58(0.39- \\
0.86)\end{array}$ & 0.005 \\
\hline \multirow[t]{6}{*}{ TERT } & rs10069690 & $n=584$ & $n=568$ & & & $n=440$ & $n=550$ & & \\
\hline & $\mathrm{CC}$ & $367(62.8)$ & 390 (68.7) & $\begin{array}{l}1.00 \text { (refer- } \\
\text { ence) }\end{array}$ & & $280(63.7)$ & 394 (71.6) & $\begin{array}{l}1.00 \text { (refer- } \\
\text { ence) }\end{array}$ & \\
\hline & $\mathrm{CT}$ & $185(31.7)$ & $166(29.2)$ & $\begin{array}{l}1.19(0.92- \\
1.53)\end{array}$ & 0.189 & $137(31.1)$ & 148 (26.9) & $\begin{array}{l}1.30(0.98- \\
1.71)\end{array}$ & 0.067 \\
\hline & $\mathrm{TT}$ & $32(5.5)$ & $12(2.1)$ & $\begin{array}{l}1.69(1.20- \\
2.37)\end{array}$ & 0.003 & $23(5.2)$ & $8(1.5)$ & $\begin{array}{l}2.02(1.34- \\
3.04)\end{array}$ & $8.0 \times 10^{-4}$ \\
\hline & $\mathrm{C}$ allele & & & $\begin{array}{l}1.00 \text { (refer- } \\
\text { ence) }\end{array}$ & & & & $\begin{array}{l}1.00 \text { (refer- } \\
\text { ence) }\end{array}$ & \\
\hline & $\mathrm{T}$ allele & & & $\begin{array}{l}1.35(1.10- \\
1.67)\end{array}$ & 0.005 & & & $\begin{array}{l}1.69(1.36- \\
2.04)\end{array}$ & $7.4 \times 10^{-4}$ \\
\hline \multirow[t]{6}{*}{ TERT } & rs2853676 & $n=584$ & $n=568$ & & & $n=440$ & $n=550$ & & \\
\hline & $\mathrm{CC}$ & 379 (64.9) & $427(75.1)$ & $\begin{array}{l}1.00 \text { (refer- } \\
\text { ence) }\end{array}$ & & $289(65.7)$ & $431(78.4)$ & $\begin{array}{l}1.00 \text { (refer- } \\
\text { ence) }\end{array}$ & \\
\hline & $\mathrm{CT}$ & 179 (30.7) & $135(23.8)$ & $\begin{array}{l}1.50(1.15- \\
1.95)\end{array}$ & 0.003 & $133(30.2)$ & $113(20.5)$ & $\begin{array}{l}1.76(1.31- \\
2.35)\end{array}$ & $1.6 \times 10^{-4}$ \\
\hline & $\mathrm{TT}$ & $26(4.5)$ & $6(1.1)$ & $\begin{array}{l}2.20(1.41- \\
3.45)\end{array}$ & $5.8 \times 10^{-4}$ & $18(4.1)$ & $6(1.1)$ & $\begin{array}{l}2.12(1.32- \\
3.38)\end{array}$ & 0.002 \\
\hline & $\mathrm{C}$ allele & & & $\begin{array}{l}1.00 \text { (refer- } \\
\text { ence) }\end{array}$ & & & & $\begin{array}{l}1.00 \text { (refer- } \\
\text { ence) }\end{array}$ & \\
\hline & $\mathrm{T}$ allele & & & $\begin{array}{l}1.65(1.32- \\
2.08)\end{array}$ & $1.6 \times 10^{-5}$ & & & $\begin{array}{l}1.76(1.45- \\
2.13)\end{array}$ & $4.9 \times 10^{-6}$ \\
\hline \multirow[t]{6}{*}{ VPS34 } & rs2162440 & $n=575$ & $n=560$ & & & $n=440$ & $n=550$ & & \\
\hline & GG & 343 (59.6) & 369 (65.9) & $\begin{array}{l}1.00 \text { (refer- } \\
\text { ence) }\end{array}$ & & $256(58.2)$ & 368 (66.9) & $\begin{array}{l}1.00 \text { (refer- } \\
\text { ence) }\end{array}$ & \\
\hline & GA & 204 (35.5) & $168(30.0)$ & $\begin{array}{l}1.31(1.02- \\
1.68)\end{array}$ & 0.037 & 157 (35.7) & $162(29.5)$ & $\begin{array}{l}1.40(1.07- \\
1.84)\end{array}$ & 0.015 \\
\hline & AA & $28(4.9)$ & $23(4.1)$ & $\begin{array}{l}1.15(0.86- \\
1.53)\end{array}$ & 0.348 & $27(6.1)$ & $20(3.6)$ & $\begin{array}{l}1.22(0.89- \\
1.68)\end{array}$ & 0.211 \\
\hline & $\mathrm{G}$ allele & & & $\begin{array}{l}1.00 \text { (refer- } \\
\text { ence) }\end{array}$ & & & & $\begin{array}{l}1.00 \text { (refer- } \\
\text { ence) }\end{array}$ & \\
\hline & A allele & & & $\begin{array}{l}1.23(1.01- \\
1.51)\end{array}$ & 0.046 & & & $\begin{array}{l}1.47(1.20- \\
1.79)\end{array}$ & 0.006 \\
\hline
\end{tabular}


Table 3 (continued)

\begin{tabular}{|c|c|c|c|c|c|}
\hline \multirow[t]{2}{*}{ Genes } & \multirow[t]{2}{*}{ SNP IDs } & \multicolumn{4}{|c|}{ Combined samples } \\
\hline & & Cases, no. (\%) & $\begin{array}{l}\text { Controls, no. } \\
(\%)\end{array}$ & $\mathrm{OR}^{1}(95 \% \mathrm{CI})$ & $P$ \\
\hline \multirow[t]{6}{*}{ CXCR4 } & rs6430612 & $n=1020$ & $n=1118$ & & \\
\hline & $\mathrm{CC}$ & $936(91.8)$ & $972(86.9)$ & $\begin{array}{l}1.00 \text { (refer- } \\
\text { ence) }\end{array}$ & \\
\hline & $\mathrm{CT}$ & $83(8.1)$ & $135(12.1)$ & $\begin{array}{l}0.64(0.78- \\
0.85)\end{array}$ & 0.002 \\
\hline & TT & $1(0.1)$ & $11(1.0)$ & $\mathrm{NC}$ & $\mathrm{NC}$ \\
\hline & $\mathrm{C}$ allele & & & $\begin{array}{l}1.00 \text { (refer- } \\
\text { ence) }\end{array}$ & \\
\hline & $\mathrm{T}$ allele & & & $\begin{array}{l}0.61(0.51- \\
0.78)\end{array}$ & $1.4 \times 10^{-4}$ \\
\hline \multirow[t]{6}{*}{$T E R T$} & rs 10069690 & $n=1024$ & $n=1118$ & & \\
\hline & $\mathrm{CC}$ & $647(63.2)$ & $784(70.1)$ & $\begin{array}{l}1.00 \text { (refer- } \\
\text { ence) }\end{array}$ & \\
\hline & CT & $322(31.4)$ & $314(28.1)$ & $\begin{array}{l}1.24(1.03- \\
1.50)\end{array}$ & 0.023 \\
\hline & TT & $55(5.4)$ & $20(1.8)$ & $\begin{array}{l}1.83(1.41- \\
2.38)\end{array}$ & $5.8 \times 10^{-6}$ \\
\hline & $\mathrm{C}$ allele & & & $\begin{array}{l}1.00 \text { (refer- } \\
\text { ence) }\end{array}$ & \\
\hline & $\mathrm{T}$ allele & & & $\begin{array}{l}1.53(1.32- \\
1.85)\end{array}$ & $4.8 \times 10^{-6}$ \\
\hline \multirow[t]{6}{*}{$T E R T$} & rs2853676 & $n=1024$ & $n=1118$ & & \\
\hline & $\mathrm{CC}$ & $668(65.2)$ & $858(76.7)$ & $\begin{array}{l}1.00 \text { (refer- } \\
\text { ence) }\end{array}$ & \\
\hline & $\mathrm{CT}$ & $312(30.5)$ & $248(22.2)$ & $\begin{array}{l}1.62(1.33- \\
1.97)\end{array}$ & $1.3 \times 10^{-6}$ \\
\hline & TT & $44(4.3)$ & $12(1.1)$ & $\begin{array}{l}2.17(1.57- \\
3.00)\end{array}$ & $2.6 \times 10^{-6}$ \\
\hline & $\mathrm{C}$ allele & & & $\begin{array}{l}1.00 \text { (refer- } \\
\text { ence) }\end{array}$ & \\
\hline & $\mathrm{T}$ allele & & & $\begin{array}{l}1.82(1.53- \\
2.01)\end{array}$ & $5.2 \times 10^{-7}$ \\
\hline \multirow[t]{6}{*}{ VPS34 } & rs2162440 & $n=1009$ & $n=1110$ & & \\
\hline & GG & $599(59.4)$ & $737(66.4)$ & $\begin{array}{l}1.00 \text { (refer- } \\
\text { ence) }\end{array}$ & \\
\hline & GA & $361(35.8)$ & $330(29.7)$ & $\begin{array}{l}1.35(1.12- \\
1.62)\end{array}$ & 0.002 \\
\hline & AA & $49(4.8)$ & $43(3.9)$ & $\begin{array}{l}1.18(0.96- \\
1.46)\end{array}$ & 0.125 \\
\hline & $\mathrm{G}$ allele & & & $\begin{array}{l}1.00 \text { (refer- } \\
\text { ence) }\end{array}$ & \\
\hline & A allele & & & $\begin{array}{l}1.07(1.19- \\
1.55)\end{array}$ & 0.004 \\
\hline
\end{tabular}

SNP single-nucleotide polymorphism, GCA gastric cardia adenocarcinoma, $N C$ not calculated, $O R$ odds ratio, $C I$ confidence interval

${ }^{1}$ Data were calculated by logistic regression with adjustment for age, sex, smoking and drinking status 
Table 4 Risk of GCA associated with CXCR4, TERT, and VPS34 SNPs by age, sex, smoking, and drinking status

\begin{tabular}{|c|c|c|c|c|c|c|c|c|c|c|}
\hline \multirow[t]{2}{*}{ Variables } & \multicolumn{5}{|c|}{ CXCR4 rs6430612 } & \multicolumn{5}{|c|}{ TERT rs 10069690} \\
\hline & $\mathrm{CC}^{\mathrm{a}}$ & $\mathrm{CT}+\mathrm{TT}^{\mathrm{a}}$ & $\mathrm{OR}^{\mathrm{b}}(95 \% \mathrm{CI})$ & $P$ & $P_{\text {interaction }}$ & $\mathrm{CC}^{\mathrm{a}}$ & $\mathrm{CT}+\mathrm{TT}^{\mathrm{a}}$ & $\mathrm{OR}^{\mathrm{b}}(95 \% \mathrm{CI})$ & $P$ & $P_{\text {interaction }}$ \\
\hline Age (year) & & & & & 0.058 & & & & & 0.080 \\
\hline$\leq 66$ & $498 / 477$ & $40 / 81$ & $0.44(0.29-0.68)$ & $1.5 \times 10^{-4}$ & & $341 / 381$ & $199 / 177$ & $1.14(0.87-1.48)$ & 0.349 & \\
\hline$>66$ & $438 / 495$ & $44 / 65$ & $0.84(0.55-1.28)$ & 0.413 & & $306 / 403$ & $178 / 157$ & $1.50(1.14-1.96)$ & 0.004 & \\
\hline Sex & & & & & 0.025 & & & & & 0.771 \\
\hline Male & $793 / 800$ & $64 / 124$ & $0.51(0.37-0.72)$ & $1.2 \times 10^{-4}$ & & $549 / 648$ & $312 / 276$ & $1.29(1.05-1.60)$ & 0.017 & \\
\hline Female & $143 / 172$ & $20 / 22$ & $0.86(0.44-1.71)$ & 0.674 & & $98 / 136$ & $65 / 58$ & $2.36(1.41-3.95)$ & 0.001 & \\
\hline Smoking status & & & & & 0.928 & & & & & $9.7 \times 10^{-5}$ \\
\hline Nonsmoker & $475 / 745$ & $47 / 116$ & $0.58(0.40-0.83)$ & 0.003 & & $319 / 632$ & $203 / 229$ & $1.78(1.40-2.26)$ & $3.3 \times 10^{-6}$ & \\
\hline Smoker & $461 / 227$ & $37 / 30$ & $0.53(0.31-0.90)$ & 0.018 & & $328 / 152$ & $174 / 105$ & $0.82(0.59-1.14)$ & 0.232 & \\
\hline Alcohol drinking & & & & & 0.937 & & & & & $4.6 \times 10^{-5}$ \\
\hline No & $534 / 627$ & $53 / 103$ & $0.60(0.42-0.85)$ & 0.005 & & $366 / 538$ & $221 / 192$ & $1.75(1.37-2.22)$ & $5.6 \times 10^{-6}$ & \\
\hline Yes & $402 / 345$ & $31 / 43$ & $0.51(0.29-0.90)$ & 0.020 & & $281 / 246$ & $156 / 142$ & $0.78(0.55-1.10)$ & 0.150 & \\
\hline \multirow[t]{2}{*}{ Variables } & \multicolumn{5}{|c|}{$T E R T$ rs 2853676} & \multicolumn{5}{|c|}{ VPS34 rs2162440 } \\
\hline & $\mathrm{CC}^{\mathrm{a}}$ & $\mathrm{CT}+\mathrm{TT}^{\mathrm{a}}$ & $\mathrm{OR}^{\mathrm{b}}(95 \% \mathrm{CI})$ & $P$ & $P_{\text {interaction }}$ & $\mathrm{GG}^{\mathrm{a}}$ & $\mathrm{GA}+\mathrm{AA}^{\mathrm{a}}$ & $\mathrm{OR}^{\mathrm{b}}(95 \% \mathrm{CI})$ & $P$ & $\overline{P_{\text {interaction }}}$ \\
\hline Age (year) & & & & & 0.156 & & & & & 0.204 \\
\hline$\leq 66$ & $361 / 445$ & $179 / 113$ & $2.01(1.51-2.69)$ & $2.3 \times 10^{-6}$ & & $307 / 372$ & $222 / 181$ & $1.39(1.07-1.80)$ & 0.015 & \\
\hline$>66$ & $307 / 413$ & $177 / 147$ & $1.68(1.28-2.22)$ & $2.1 \times 10^{-4}$ & & $292 / 365$ & $188 / 192$ & $1.24(0.95-1.61)$ & 0.114 & \\
\hline Sex & & & & & 0.335 & & & & & 0.861 \\
\hline Male & $556 / 698$ & $305 / 226$ & $1.80(1.44-2.24)$ & $1.9 \times 10^{-7}$ & & $503 / 608$ & $343 / 309$ & $1.31(1.06-1.61)$ & 0.011 & \\
\hline Female & $112 / 160$ & $51 / 34$ & $2.27(1.30-3.95)$ & 0.004 & & $96 / 129$ & $67 / 64$ & $1.45(0.90-2.34)$ & 0.123 & \\
\hline Smoking status & & & & & 0.863 & & & & & 0.332 \\
\hline Nonsmoker & $333 / 660$ & $189 / 201$ & $1.90(1.48-2.44)$ & $4.5 \times 10^{-7}$ & & $305 / 575$ & $210 / 280$ & $1.41(1.12-1.79)$ & 0.004 & \\
\hline Smoker & $335 / 198$ & $167 / 59$ & $1.58(1.10-2.28)$ & 0.014 & & $294 / 162$ & $200 / 93$ & $1.24(0.89-1.73)$ & 0.202 & \\
\hline Alcohol drinking & & & & & 0.881 & & & & & 0.126 \\
\hline No & $382 / 555$ & $205 / 175$ & $1.85(1.44-2.36)$ & $1.1 \times 10^{-6}$ & & $334 / 487$ & $246 / 238$ & $1.48(1.18-1.87)$ & 0.001 & \\
\hline Yes & $286 / 303$ & $151 / 85$ & $1.65(1.13-2.40)$ & 0.010 & & $265 / 250$ & $164 / 135$ & $1.05(0.74-1.49)$ & 0.772 & \\
\hline
\end{tabular}

${ }^{a}$ Number of case patients with the genotype/number of control subjects with the genotype(s)

${ }^{\mathrm{b}}$ Data were calculated by logistic regression, adjusted for sex, age, smoking and drinking status, where it was appropriate

95\% CI 1.45-2.11; VPS34 rs2162440: OR 1.39, 95\% CI $1.16-1.65)$.

\section{Stratified analyses of associations between CXCR4, TERT and VPS34 polymorphisms and GCA risk}

The risk of GCA associated with the CXCR4, TERT and VPS34 polymorphisms was further examined by stratifying for age, sex, smoking and alcohol drinking status using the combined data of two case-control sets (Table 4). For the CXCR4 SNP, a significantly reduced risk of GCA associated with the rs6430612 CT or TT genotype compared with the CC genotype was observed for the group aged 66 years or younger (OR $0.44,95 \%$ CI $0.29-0.68, P=1.5 \times 10^{-4}$ ), but not for the group aged older than 66 years $(P=0.413)$. Compared with the CC genotype, a decreased risk of GCA was only associated with $C X C R 4 \mathrm{CT}$ or TT genotype for the male group (OR $0.51,95 \%$ CI $0.37-0.72, P=1.2 \times 10^{-4}$ ), but not among female subjects $(P=0.674)$. There was statistically significant gene-age interaction for the $C X C R 4 \mathrm{SNP}$ $\left(P_{\text {interaction }}=0.025\right)$. In stratified analyses with smoking or alcohol drinking status, the CXCR4 rs6430612 polymorphism was significantly associated with decreased risk in smokers, nonsmokers, drinkers, or nondrinkers (all $P<0.05$ ) (Table 4).

For the TERT rs 10069690 genetic variant, carriers of the rs10069690 CT or TT genotype and aged older than 66 years showed significantly elevated risk to develop GCA compared with the rs $10069690 \mathrm{CC}$ carriers (OR $1.50,95 \%$ CI $1.14-1.96, P=0.004)$. However, no such association was found for the group aged 66 years or younger $(P=0.349)$. It was observed that an increased risk of GCA was associated with the rs 10069690 genotype in both males and females (both $P<0.05$ ). Stratified analyses with smoking or drinking status showed that significantly elevated ORs for GCA development 
were found in nonsmokers (OR 1.78, 95\% CI 1.40-2.26, $P=3.3 \times 10^{-6}$ ) as well as nondrinkers (OR $1.75,95 \% \mathrm{CI}$ 1.37-2.22, $\left.P=5.6 \times 10^{-6}\right)$. However, there was no significantly increased risk for smokers or drinkers with the rs10069690 CT or TT genotype compared with smokers or drinkers with the CC genotype $(P=0.232, P=0.150)$. A statistically significant gene-smoking or gene-drinking interaction was observed $\left(P_{\text {interaction }}=9.7 \times 10^{-5}\right.$ or $\left.4.6 \times 10^{-5}\right)$. For the TERT rs $2853676 \mathrm{SNP}$, significant associations between the rs $2853676 \mathrm{CT}$ or TT genotype and GCA risk was found in all stratified analyses with age, sex, smoking and alcohol drinking status (all $P<0.05$ ) (Table 4).

For the VPS34 rs 2162440 polymorphism, stratified analyses showed that significantly increased ORs for GCA development were only observed in the group aged 66 years or younger (OR 1.39, 95\% CI 1.07-1.80, $P=0.015$ ), males (OR 1.31, 95\% CI 1.06-1.61, $P=0.011$ ), nonsmokers (OR 1.41, 95\% CI 1.12-1.79, $P=0.004$ ), as well as nondrinkers (OR 1.48, $95 \%$ CI $1.18-1.87, P=0.001)$. No statistically significant gene-environment interactions were observed between the $V P S 34 \mathrm{SNP}$ and the above-mentioned factors (all $P_{\text {interaction }}$ $>0.05)$.

\section{Discussion}

Globally, gastric cancer incidence shows diverse characteristics by the two major topographical subsites, GCA, and noncardia gastric cancer [3]. GCA has distinct epidemiological, histopathological, and molecular characteristics which distinguish it from the adenocarcinomas of distal stomach [3-8]. Telomere shortening, in the course of somatic cell replication, ultimately results in replicative senescence and multiple aging-related complex genetic diseases. We previously found that shortened leukocyte telomere length significantly contributes to increased risk of GCA [10]. In humans, various genetic variations have been identified to be associated with leukocyte telomere length via GWAS [20-29] and most of these polymorphisms have been proved to confer cancer susceptibility. In the current study, we, for the first time, systematically evaluate the involvement of these telomere length-related genetic variations in GCA development. We found that CXCR4 rs6430612, TERT rs10069690 and rs2853676, as well as VPS34 rs2162440 polymorphisms significantly contribute to GCA risk in different Chinese populations.

CXCR4, the receptor of chemokine CXCL12, plays an important role in gastric cancer. Overexpressed CXCR4 was associated with more advanced tumor stage and poorer survival for gastric cancer patients [33]. CXCR4 can activate either the NF- $\kappa \mathrm{B} / \mathrm{STAT} 3$ signaling or the $\mathrm{PI} 3 \mathrm{~K} / \mathrm{AKT} / \mathrm{mTOR}$ signaling, while NF- $\mathrm{BBp} 65$ can then transcriptionally activate CXCR4 [33-35]. CXCR4-mediated invasion can be regulated by HER2, CD44, miR-139, and DARPP-32 in gastric cancer cells [36, 37]. CXCL12/ CXCR4 signaling activates leukocytes and is often induced by proinflammatory stimuli, which can partially illuminate the association between rs6430612 and telomere length as well as GCA risk.

Human TERT is essential for the maintenance of telomere length, chromosomal stability, and cellular immortality. Accumulated evidences demonstrated that various TERT SNPs including rs10069690 and rs2853676 are associated with multiple cancer types [25, 38-44]. Duan et al. reported in a case-control study enrolled a total of 302 patients and 300 control individuals, and found that both rs 10069690 and rs 2853676 polymorphisms were significantly associated with gastric cancer [41]. However, they did not show the detailed information of gastric cancer patients whether or not including GCA. In the present study with 1024 GCA patients and 1118 controls, we, for the first time, demonstrated that a significantly increased GCA risk was associated with minor alleles of both SNPs (both $P<10^{-5}$ ). For the detailed mechanistic insights into these GCA risk-associated SNPs, Killedar et al. found that the rs $10069690 \mathrm{~T}$ allele creates an additional splice donor site in intron 4 of TERT, and results in co-production of full-length TERT and an alternatively spliced, INS 1b, transcript [38]. INS 1b protein does not have telomerase enzyme activity, but retains its ability to bind to the telomerase RNA subunit. This leads to decreased telomerase activity and telomere shortening [38]. These data are in line to our findings that the rs $10069690 \mathrm{~T}$ allele is a GCA risk allele associated with short telomere length.

In a previous study, Mangino et al. conducted a GWAS of 314,075 SNPs and validated the results in a second cohort ( $n$ for both cohorts combined $=2790$ ) and identified VPS34 rs2162440 as a novel leukocyte telomere length-associated variants [27]. The involvement of rs2162440 in cancer development is unclear and we provided the first detailed data on its significant contribution to GCA risk. As a component of the PI3 kinase family, VPS34 regulates multiple aspect of the cell physiology [45]. Interestingly, Vps34, the VPS34 yeast orthologue, is directly involved in the pathway controlling telomere length variation [46]. As a result, it is possible that the VPS34 rs2162440 may impact telomere maintenance, lead to an increased risk of genetic instability and therefore of tumorigenesis.

In all, to our knowledge, we, for the first time, identified four leukocyte telomere length-related genetic variants that significantly confer susceptibility to GCA. These results are consistent with our initial findings on association between leukocyte telomere length and GCA risk, further supporting the importance of telomere biology during carcinogenesis. However, some limitations exist in this case-control study. 
For example, there might be inherent selection bias, since all GCA cases were recruited from hospitals. As a result, our findings warrant to be validated in a population-based prospective study in the future.

Acknowledgements This study was supported by National Natural Science Foundation of China (31671300, 31871306); Taishan Scholars Program of Shandong Province (tsqn20161060).

\section{Compliance with ethical standards}

Conflict of interest The authors declare no competing financial interests.

Human rights All procedures followed were in accordance with the ethical standards of the responsible committee on human experimentation (institutional and national) and with the Helsinki Declaration of 1964 and later versions. Informed consent to be included in the study was obtained from all individuals.

\section{References}

1. Torre LA, Bray F, Siegel RL, Ferlay J, Lortet-Tieulent J, Jemal A. Global cancer statistics, 2012. CA Cancer J Clin. 2015;65:87-108.

2. Chen W, Zheng R, Zhang S, Zeng H, Xia C, Zuo T, et al. Cancer incidence and mortality in China, 2013. Cancer Lett. 2017;401:63-71.

3. Colquhoun A, Arnold M, Ferlay J, Goodman KJ, Forman D, Soerjomataram I. Global patterns of cardia and non-cardia gastric cancer incidence in 2012. Gut. 2015;64:1881-8.

4. Heidl G, Langhans P, Mellin W, Bunte H, Grundmann E. Adenocarcinomas of esophagus and cardia in comparison with gastric carcinoma. J Cancer Res Clin Oncol. 1993;120:95-9.

5. Bahmanyar S, Ye W. Dietary patterns and risk of squamous-cell carcinoma and adenocarcinoma of the esophagus and adenocarcinoma of the gastric cardia: a population-based case-control study in Sweden. Nutr Cancer. 2006;54:171-8.

6. He YT, Hou J, Chen ZF, Qiao CY, Song GH, Meng FS, et al. Trends in incidence of esophageal and gastric cardia cancer in high-risk areas in China. Eur J Cancer Prev. 2008;17:71-6.

7. Mayne ST, Risch HA, Dubrow R, Chow WH, Gammon MD, Vaughan TL, et al. Nutrient intake and risk of subtypes of esophageal and gastric cancer. Cancer Epidemiol Biomark Prev. 2001;10:1055-62.

8. Ye W, Chow WH, Lagergren J, Yin L, Nyren O. Risk of adenocarcinomas of the esophagus and gastric cardia in patients with gastroesophageal reflux diseases and after antireflux surgery. Gastroenterology. 2001;121:1286-93.

9. Hu N, Wang Z, Song X, Wei L, Kim BS, Freedman ND, et al. Genome-wide association study of gastric adenocarcinoma in Asia: a comparison of associations between cardia and noncardia tumours. Gut. 2016;65:1611-8.

10. Liu Y, Lei T, Zhang N, Zheng Y, Kou P, Shang S, et al. Leukocyte telomere length and risk of gastric cardia adenocarcinoma. Sci Rep. 2018;8:14584.

11. Wang Z, Dai J, Hu N, Miao X, Abnet CC, Yang M, et al. Identification of new susceptibility loci for gastric non-cardia adenocarcinoma: pooled results from two Chinese genome-wide association studies. Gut. 2017;66:581-7.

12. Yang M, Guo Y, Zhang X, Miao X, Tan W, Sun T, et al. Interaction of P53 Arg72Pro and MDM2 T309G polymorphisms and their associations with risk of gastric cardia cancer. Carcinogenesis. 2007;28:1996-2001.

13. Blackburn EH. Telomeres and telomerase: the means to the end (Nobel lecture). Angew Chem Int Ed Engl. 2010;49:7405-21.

14. Zakian VA. Telomeres: beginning to understand the end. Science. 1995;270:1601-7.

15. Olovnikov AM. A theory of marginotomy. The incomplete copying of template margin in enzymic synthesis of polynucleotides and biological significance of the phenomenon. $\mathrm{J}$ Theor Biol. 1973;41:181-90.

16. Valdes AM, Andrew T, Gardner JP, Kimura M, Oelsner E, Cherkas LF, et al. Obesity, cigarette smoking, and telomere length in women. Lancet. 2005;366:662-4.

17. Aviv A. Telomeres and human somatic fitness. J Gerontol A Biol Sci Med Sci. 2006;61:871-3.

18. Brown WR. Molecular cloning of human telomeres in yeast. Nature. 1989;338:774-6.

19. de Lange T. Cell biology. Telomere capping-one strand fits all. Science. 2001;292:1075-6.

20. Ojha J, Codd V, Nelson CP, Samani NJ, Smirnov IV, Madsen NR, et al. Genetic variation associated with longer telomere length increases risk of chronic lymphocytic leukemia. Cancer Epidemiol Biomark Prev. 2016;25:1043-9.

21. Julin B, Shui I, Heaphy CM, Joshu CE, Meeker AK, Giovannucci $\mathrm{E}$, et al. Circulating leukocyte telomere length and risk of overall and aggressive prostate cancer. $\mathrm{Br} \mathrm{J}$ Cancer. 2015;112:769-76.

22. Levy D, Neuhausen SL, Hunt SC, Kimura M, Hwang SJ, Chen $\mathrm{W}$, et al. Genome-wide association identifies OBFC1 as a locus involved in human leukocyte telomere biology. Proc Natl Acad Sci USA. 2010;107:9293-8.

23. Shen Q, Zhang Z, Yu L, Cao L, Zhou D, Kan M, et al. Common variants near TERC are associated with leukocyte telomere length in the Chinese Han population. Eur J Hum Genet. 2011;19:721-3.

24. Jones AM, Beggs AD, Carvajal-Carmona L, Farrington S, Tenesa A, Walker M, et al. TERC polymorphisms are associated both with susceptibility to colorectal cancer and with longer telomeres. Gut. 2012;61:248-54.

25. Terry KL, Tworoger SS, Vitonis AF, Wong J, Titus-Ernstoff L, De Vivo I, et al. Telomere length and genetic variation in telomere maintenance genes in relation to ovarian cancer risk. Cancer Epidemiol Biomark Prev. 2012;21:504-12.

26. Codd V, Mangino M, van der Harst P, Braund PS, Kaiser M, Beveridge AJ, et al. Common variants near TERC are associated with mean telomere length. Nat Genet. 2010;42:197-9.

27. Mangino M, Richards JB, Soranzo N, Zhai G, Aviv A, Valdes AM, et al. A genome-wide association study identifies a novel locus on chromosome 18q12.2 influencing white cell telomere length. J Med Genet. 2009;46:451-4.

28. Concetti F, Carpi FM, Nabissi M, Picciolini M, Santoni G, Napolioni V. The functional polymorphism rs73598374:G> A (p.Asp8Asn) of the ADA gene is associated with telomerase activity and leukocyte telomere length. Eur J Hum Genet. 2015;23:267-70.

29. Walsh KM, Codd V, Smirnov IV, Rice T, Decker PA, Hansen $\mathrm{HM}$, et al. Variants near TERT and TERC influencing telomere length are associated with high-grade glioma risk. Nat Genet. 2014;46:731-5.

30. Yuan J, Zhang N, Yin L, Zhu H, Zhang L, Zhou L, et al. Clinical implications of the autophagy core gene variations in advanced lung adenocarcinoma treated with Gefitinib. Sci Rep. 2017;7:17814.

31. Yuan J, Zhang N, Zhu H, Liu J, Xing H, Ma F, et al. CHST9 rs1436904 genetic variant contributes to prognosis of triple-negative breast cancer. Sci Rep. 2017;7:11802. 
32. Shi M, Ma F, Liu J, Xing H, Zhu H, Yu J, et al. A functional BRCA1 coding sequence genetic variant contributes to prognosis of triple-negative breast cancer, especially after radiotherapy. Breast Cancer Res Treat. 2017;166:109-16.

33. Xiang Z, Zhou ZJ, Xia GK, Zhang XH, Wei ZW, Zhu JT, et al. A positive crosstalk between CXCR 4 and CXCR2 promotes gastric cancer metastasis. Oncogene. 2017;36:5122-33.

34. Hashimoto I, Koizumi K, Tatematsu M, Minami T, Cho S, Takeno $\mathrm{N}$, et al. Blocking on the CXCR4/mTOR signalling pathway induces the anti-metastatic properties and autophagic cell death in peritoneal disseminated gastric cancer cells. Eur J Cancer. 2008;44:1022-9.

35. Chen G, Chen SM, Wang X, Ding XF, Ding J, Meng LH. Inhibition of chemokine (CXC motif) ligand 12/chemokine (CXC motif) receptor 4 axis (CXCL12/CXCR4)-mediated cell migration by targeting mammalian target of rapamycin (mTOR) pathway in human gastric carcinoma cells. J Biol Chem. 2012;287:12132-41.

36. Bao W, Fu HJ, Xie QS, Wang L, Zhang R, Guo ZY, et al. HER2 interacts with CD44 to up-regulate CXCR4 via epigenetic silencing of microRNA-139 in gastric cancer cells. Gastroenterology. 2011;141:2076-87 e6.

37. Zhu S, Hong J, Tripathi MK, Sehdev V, Belkhiri A, El-Rifai W. Regulation of CXCR4-mediated invasion by DARPP-32 in gastric cancer cells. Mol Cancer Res. 2013;11:86-94.

38. Killedar A, Stutz MD, Sobinoff AP, Tomlinson CG, Bryan TM, Beesley J, et al. A common cancer risk-associated allele in the hTERT locus encodes a dominant negative inhibitor of telomerase. PLoS Genet. 2015;11:e1005286.

39. Bojesen SE, Pooley KA, Johnatty SE, Beesley J, Michailidou K, Tyrer JP, et al. Multiple independent variants at the TERT locus are associated with telomere length and risks of breast and ovarian cancer. Nat Genet. 2013;45:371-84, 84e1-2.

40. Speedy HE, Di Bernardo MC, Sava GP, Dyer MJ, Holroyd A, Wang Y, et al. A genome-wide association study identifies multiple susceptibility loci for chronic lymphocytic leukemia. Nat Genet. 2014;46:56-60.

41. Duan X, Cao W, Wang L, Liu S, Liu Z, Zhang B, et al. Genetic variants in TERT are associated with risk of gastric cancer in a Chinese Han population. Oncotarget. 2016;7:82727-32.

42. Wu Y, Yan M, Li J, Li J, Chen Z, Chen P, et al. Genetic polymorphisms in TERT are associated with increased risk of esophageal cancer. Oncotarget. 2017;8:10523-30.

43. Gudmundsson J, Thorleifsson G, Sigurdsson JK, Stefansdottir L, Jonasson JG, Gudjonsson SA, et al. A genome-wide association study yields five novel thyroid cancer risk loci. Nat Commun. 2017;8:14517.

44. Cao JL, Yuan P, Abuduwufuer A, Lv W, Yang YH, Hu J. Association between the TERT genetic polymorphism rs2853676 and cancer risk: meta-analysis of 76,108 cases and 134,215 controls. PLoS One. 2015;10:e0128829.

45. Volinia S, Dhand R, Vanhaesebroeck B, MacDougall LK, Stein R, Zvelebil MJ, et al. A human phosphatidylinositol 3-kinase complex related to the yeast Vps34p-Vps15p protein sorting system. EMBO J. 1995;14:3339-48.

46. Rog O, Smolikov S, Krauskopf A, Kupiec M. The yeast VPS genes affect telomere length regulation. Curr Genet. 2005;47:18-28.

Publisher's Note Springer Nature remains neutral with regard to jurisdictional claims in published maps and institutional affiliations. 